\title{
ANÁLISE DE UM CURSO ONLINE SOBRE TUBERCULOSE NA PERSPECTIVA DE ESTUDANTES E PROFISSIONAIS DE ENFERMAGEM
}

DOI: 10.48075/ri.v24i1.28579

\author{
Thaís Tânia Ávila ${ }^{1}$ \\ Fábio Junior Martins ${ }^{2}$ \\ Fernanda Carminati de Moura ${ }^{3}$ \\ Maria de Lourdes de Almeida ${ }^{4}$ \\ Nathalia Halax Orfão ${ }^{5}$ \\ Maria Luzia Topanotti ${ }^{6}$
}

RESUMO: O estudo teve o objetivo de analisar a opinião de estudantes formandos e profissionais de enfermagem sobre um curso de capacitação em tuberculose no ambiente da Plataforma Moodle. Estudo exploratório e abordagem qualitativa realizado a partir de um curso online em tuberculose com 12 participantes, em outubro de 2020. Utilizou-se a técnica de Grupo Focal online, baseado em questões norteadoras voltadas ao curso, e a Análise de Conteúdo de Minayo. Os relatos analisaram o curso em diversos aspectos, classificando-o de forma positiva, os participantes destacaram que a maioria das pessoas buscam atualização profissional valorizando métodos de ensino que utilizam inovações tecnológicas, as quais quando aliadas a teoria e prática no formato educativo permitem sanar dúvidas como por exemplo quanto a tuberculose. As estratégias de controle da tuberculose ainda carecem de uma dimensão integral de assistência, e para tal, ações educativas são fundamentais, sendo que a modalidade a distância possibilita o acesso a novos conhecimentos de maneira mais democrática.

Palavras-chave: Educação a Distância, Educação Permanente em Saúde, Tuberculose.

\footnotetext{
1 Bacharel e Licenciatura em enfermagem, Mestranda em Ensino pela Universidade Estadual do Oeste do Paraná (UNIOESTE). E-mail thaist avila@hotmail.com

2 Bacharel em Ciências da Computação. Mestranda em Ensino pela Universidade Estadual do Oeste do Paraná (UNIOESTE). E-mail martins.fabiojr@gmail.com

3 Bacharel e Licenciatura em enfermagem, Mestre em Ensino pela Universidade Estadual do Oeste do Paraná (UNIOESTE). E-mail enffercarminati@hotmail.com

4 Bacharel em Enfermagem, Doutora em Enfermagem, Docente da Universidade Estadual do Oeste do Paraná (UNIOESTE). E-mail m lourdesdealmeida@yahoo.com.br

5 Bacharel em Enfermagem, Doutora em Ciências, Docente da Fundação Universidade Federal de Rondônia (UNIR). E-mail nathaliahalax@unir.br

${ }^{6}$ Médica da Secretaria de Saúde de Foz do Iguaçu. E-mail malutopa@hotmail.com
} 


\section{ANALYSIS OF AN ONLINE COURSE ON TUBERCULOSIS FROM THE PERSPECTIVE OF STUDENTS AND NURSING PROFESSIONALS}

ABSTRACT: The study aimed to analyze the opinion of undergraduate students and nursing professionals about a training course on tuberculosis in the Moodle Platform environment. Exploratory study and qualitative approach carried out from an online course in tuberculosis with 12 participants, in October 2020. The Focus Group online technique was used, based on guiding questions focused on the course, and the Minayo's Content Analysis. The reports analyzed the course in various aspects, classifying it positively, the participants highlighted that most people seek professional updating, valuing teaching methods that use technological innovations, which when combined with theory and practice in the educational format, allow to resolve doubts as for example regarding tuberculosis. Tuberculosis control strategies still lack an integral dimension of care, and for that, educational actions are essential, and the distance modality allows access to new knowledge in a more democratic way.

Key Words: Distance Education, Permanent Health Education, Tuberculosis.

\section{INTRODUÇÃO}

A Tuberculose (TB) é um grave problema de saúde pública e uma das principais doenças infecciosas no mundo. A estimava global, aponta 10 milhões de casos novos e aproximadamente 1,4 milhão foram a óbito, em 2019, mesmo sendo uma doença tratável e curável (WHO, 2020). No Brasil, estes números chegam a 73.864 casos novos notificados e 4.532 tiveram o óbito como desfecho (BRASIL, 2020; 2021; WHO, 2020).

Neste sentido, visando as ações de controle da TB, torna-se necessário reforçar as atribuições e responsabilidades da Atenção Primária à Saúde (APS), a qual inclui a busca de sintomáticos respiratórios, realização de exames para diagnóstico da doença, fluxo eficiente com os laboratórios, Tratamento Diretamente Observado (TDO) e investigação dos contatos (BRASIL, 2019).

Entretanto, a falta de capacitação dos profissionais de saúde é um dos aspectos que dificultam o controle da TB (CAMPOS, 2016), sendo que a utilização de diferentes modalidades de ensino-aprendizagem, tais como Educação à distância (EaD) podem contribuir com o desenvolvimento das estratégias de Educação Permanente em Saúde (EPS) na APS, oferecendo recursos que inovam no cuidado por meio da qualificação dos profissionais e interligando os pontos necessários às estratégias de melhoria, monitoramento e controle da TB (WYSOCKI et al., 2017). 
Assim, este estudo teve como objetivo analisar a opinião de estudantes formandos e profissionais de enfermagem sobre um curso online de capacitação disponibilizado na plataforma Modular Object-Oriented Dynamic Learning Environment (Moodle) sobre TB.

\section{MATERIAIS E MÉTODOS}

Trata-se de um estudo exploratório e abordagem qualitativa realizado com seis formandos do curso de Enfermagem da Universidade Estadual do Oeste do Paraná (UNIOESTE), campus Foz do Iguaçu, Paraná, e seis enfermeiros que atuam na APS deste mesmo município. Considerou-se apenas os formandos desta instituição devido o curso ter carga horária integral na modalidade bacharelado e licenciatura.

O convite foi realizado por meio de e-mail, sendo que aqueles que aceitaram o convite foram inscritos na Plataforma Moodle, também contida no servidor do Laboratório de Epidemiologia e Estudos Operacionais em Saúde (LEO) para acesso ao curso de TB (http://leo.kinghost.net/ensino/login/index.php), entre 01 a 16 de outubro de 2020.

A coleta de dados ocorreu após a realização do curso, utilizando-se do desenvolvimento de dois grupos focais (GF) online por meio da plataforma Google Meet, sendo um grupo com os formandos e outro com os profissionais. Cada grupo contava com a presença de um moderador e um observador, com agendamento em datas distintas. Para esclarecimento de eventuais dúvidas foi disponibilizado um e-mail pessoal e o contato telefônico para suporte.

Os GF é uma técnica derivada de entrevistas grupais, que coleta informações por meio das interações grupais, e de acordo com os objetivos da investigação, cabe a criação de um ambiente favorável à discussão que propicie aos participantes manifestar suas percepções e pontos de vista (MINAYO, 2007). O que difere um GF de um GF online é unicamente o fato de este último ser realizado em um ambiente não presencial, dispensando a presença física dos participantes, considerada uma das principais vantagens, principalmente durante a pandemia da Covid-19, tal como ocorreu neste estudo.

Nos GF, foram inseridas sete perguntas norteadoras como disparadoras para a coleta de dados, e cada participante teve a sua vez de fala no processo, obtendo-se um tempo médio de 50 minutos cada GF online. Posteriormente, foi realizada a transcrição das gravações das entrevistas, leitura exaustiva e compreensiva do material transcrito, 
elaboração do agrupamento de trechos para análise, sínteses compreensivas e interpretativas, as quais foram apresentadas em categorias.

No momento da transcrição das falas e análise dos dados dos GF online, os participantes foram reclassificados, sendo diferenciados como E para os Estudantes e P para os Profissionais, seguidos do número de ordem alfabética, ficando a seguinte classificação para os estudantes (E1, E2, E3, E4, E5 e E6) e para os profissionais (P1, P2, P3, P4, P5 e P6).

As transcrições foram analisadas por meio da Análise de Conteúdo proposto por Minayo, que tem como determinadas características metodológicas: "a objetividade, sistematização e inferência, visando verificar hipóteses e/ou descobrir o que está por trás de cada conteúdo manifesto" (MINAYO, 2003, p. 74).

O projeto de pesquisa foi submetido à análise e aprovado pelo Comitê de Ética em Pesquisa da UNIOESTE, sendo aprovado sob o Certificado de Apresentação de Apreciação Ética no (15675619.4.0000.0107). Ressalta-se que todos os participantes assinaram o Termo de Consentimento Livre e Esclarecido online, conforme determina a Resolução 466/2012 do Conselho Nacional de Saúde.

\section{RESULTADOS E DISCUSSÃO}

Pelo estudo originou-se quatro categorias a partir dos GF online, sendo elas: Categoria I - Motivação para adesão a cursos online, Categoria II - Experiência de acesso e navegação no curso proposto, Categoria III - Experiência na abordagem de ensino, conteúdo, método de avaliação e linguagem do curso proposto, e, por fim, Categoria IV Potencialidades e debilidades encontradas no curso proposto.

\section{Categoria I - Motivação para adesão a cursos online}

No que se referem aos componentes motivadores na busca por cursos online em relação à temática $\mathrm{TB}$, alguns participantes relataram a busca por aprimoramento para garantir aperfeiçoamento pessoal e profissional.

[...] aprimorar nossos conhecimentos e estarmos inseridos no ambiente tecnológico, possibilitando o aperfeiçoamento pessoal e profissional (E1).

[...] aperfeiçoamento profissional, já que faz parte rotineiramente do processo de trabalho o atendimento (P3). 
Cursos no formato EaD possibilitam alcançar um maior número de profissionais, incluindo aqueles da área da saúde, que por muitas vezes precisam ou buscam aprimorar-se, sendo esta oportunizada pelo meio online que possibilita o acesso a diversos conteúdos e execução no horário mais conveniente, sem que seja necessário deslocamento para participação presencial (RANGEL, 2011), além de contribuir no processo ativo e de criticidade da realidade vivenciada por estes profissionais (MARCHISOTTI; OLIVEIRA; LUKOSEVICIUS, 2017).

[...] os cursos são sempre necessários para estarmos atualizados sobre como podemos melhorar nosso atendimento (E5).

[...] é importante estar dentro dessas novas atualizações até para conseguir ofertar melhor o tratamento para o paciente, o melhor atendimento, o melhor serviço (P2).

[...] atualizar o conhecimento, é quando eu estou por dentro do que tem de novo que isso me dá segurança pra manejar o cuidado com esse paciente (P4).

Pontua-se que através dessa busca por atualizações, as inovações tecnológicas tomaram espaço no que tange aos cursos online, principalmente no modelo de EaD, porque possui características que possibilitam o acesso a novos conhecimentos de forma igualitária, tornando o estudo e o tempo dedicado a esses flexíveis, variando de acordo com a vida pessoal de cada um e se encaixando em uma rotina (KARPINSKI et al., 2017).

Outros participantes, no entanto, buscaram estar presentes no processo de EPS, visando repassar a sua equipe um conhecimento atualizado, à procura por um melhor atendimento ao cliente, contando assim com um trabalho multidisciplinar.

[...] para o processo de educação permanente da minha equipe posteriormente, no compartilhamento desses conhecimentos para prevenção de doenças e recuperação, visando uma deteç̧ão precoce e um bom prognóstico (E6).

Martins-Melo et al. (2014) corrobora com o Ministério da Saúde e o da Educação, os quais buscam renovação nos caminhos de formação da APS que visem o serviço e ensino englobados, e que impactem de forma positiva no trabalho dos profissionais, e por consequência melhorando o atendimento ao usuário do serviço de saúde.

Em se tratando do reconhecimento desses aspectos, um estudo realizado no município de Foz do Iguaçu apontou que os enfermeiros atuantes na APS enfrentam dificuldades no que diz respeito ao conhecimento sobre o manejo da doença TB, por exemplo (SILVA-SOBRINHO et al., 2014). Nessa perspectiva, a EPS é mencionada como 
política e fator essencial para ocorrer transformações nesse contexto, além de melhorar a organização do trabalho mediante levantamento dos problemas e sugerindo maneiras de enfrentar tais percalços (FALKENBERG et al., 2014), capacitando os profissionais para um cuidado de qualidade, garantindo segurança e autonomia aos trabalhadores e à população em geral.

Reforçando essa ideia, tem-se o estudo de Pereira et al. (2016), o qual aponta que o ingresso a novas tecnologias para o desenvolvimento da EPS renova o quadro de educação e treinamento dos profissionais. Todavia, Wanderley et al. (2018) apontam algumas barreiras, tais como resistência dos facilitadores, escassez de atualização profissional e ausência desses conteúdos no processo de formação acadêmica ainda na graduação.

Coelho e Tedesco (2017), contudo, defendem as potencialidades do uso da tecnologia digital, a qual permite a expansão do espaço de armazenamento e memorização de informações, maior abrangência do processo de comunicação, além de contemplarem a interação, uma vez que estreitam as relações independentemente da localização geográfica, disseminando saberes e culturas.

\section{Categoria II - Experiência de acesso e navegação no curso proposto}

Os Ambientes Virtuais de Aprendizagem (AVA) possibilitam ampliar o acesso a capacitações, pois abrangem diversas mídias e objetos de conhecimento, o que os torna acessíveis, dinâmicos e em acordo com a realidade do trabalhador (FILATRO; CAIRO, 2015).

No que tange à experiência quanto ao acesso e navegação no curso online, a maioria dos participantes relatou que ambos são simples, objetivos, fáceis e rápidos, sendo uma experiência positiva ao usuário.

[...] fácil, mais fácil do que os outros cursos que eu já fiz também de forma online na parte de navegação, o conteúdo está bem explicado, tem vídeos, e tem os conteúdos escritos, é fácil de entender, é uma plataforma bem simples (E3).

[...] simples de acessar e também uma das coisas que achei interessante foram as opções das aulas tanto em vídeo quanto em escritas que pode facilitar no entendimento das pessoas tanto aquelas que tem facilidade em aprender por vídeo, quanto lendo. Sem contar que é um curso objetivo que pode ser realizado de forma simples (E5).

[...] prática e de fácil entendimento, achei muito interessante a disposição dos conteúdos, métodos de abordagem e avaliações também (E6). 
[...] fácil de acessar, conteúdos bem dispostos e organizados de uma forma fácil também de encontrar então quando eu entrava em um conteúdo eu tinha facilidade de voltar por exemplo (P4).

[...] navegação bem simples, a disposição dos conteúdos, achei legal que tem do ladinho pra você ir marcando o conteúdo que você já assistiu, para não repetir, para saber onde você parou, os vídeos também carregam de uma forma bem fácil, não são vídeos que demandam muito da internet para funcionar (P5).

[...] didático, bem legal, fácil da pessoa se achar ali, e ir procurando (P6).

O Moodle permite o uso de diferentes recursos tecnológicos, o qual o torna interativo e moderno, permitindo fácil acesso e maior compreensão para inserção de dados e sua disponibilização ao usuário (AVELINO et al., 2017). Possui comunicação móvel, ou seja, permite maior acessibilidade, além de incentivar uma aprendizagem autônoma e atualizada.

Ao mesmo tempo, em que exige mudanças dos participantes como sujeito ativo deste processo de aprendizagem (OLIVEIRA et al., 2019), destaca-se que a interface deve ser fácil e intuitiva (COELHO; TEDESCO, 2017), assim como foi verificado neste estudo.

Categoria III - Experiência quanto a abordagem de ensino, conteúdo, método de avaliação e linguagem do curso proposto

Quanto a abordagem de ensino, verificou-se um misto de interpretações, em geral mista e específica, com relação a esse aspecto pelos participantes.

[...] ela deixa mais amplo e também facilita para entender, se for muito específico quem for ler não vai entender né, e se for geral falando um pouquinho da doença, falando tudo, eu acho que facilita mais o entendimento (E1).

[...] abordagem geral porque abrange uma gama maior de profissionais (P6).

Os recursos tecnológicos como o EaD possibilitam a reflexão dos participantes de curso online ao promoverem diálogo e autonomia, além de estimularem a permanecer no AVA, reduzindo, assim, as evasões. Mais do que simplesmente agradar os participantes, o caminho é utilizar uma diversidade de formatos de conteúdos pela combinação de mídias (visuais e auditivas) na apresentação dos conteúdos e propor um conjunto variado de atividades, a fim de atender a um maior número de preferência, ou seja, levar em consideração a forma de aprendizagem de cada aluno (FILATRO; CAIRO, 2015), como apontam as falas dos participantes do curso em estudo. 
[...] acho que tem que ser meio a meio, uma parte geral, mais ampla, retomada de conhecimento, e outra mais específica, porque tem os iniciantes, e tem os que já estão lidando rotineiramente com isso (E2).

[...] as duas formas pois no geral você abrange todos os públicos e no específico você colabora e contribui com determinadas demandas (E6).

[...] Acredito que ambos são necessários, tanto a abordagem mais específica com enfoque nas dificuldades para aqueles profissionais que já estão na rede há mais tempo e também acho importante quanto os conceitos básicos, já que sempre há profissionais novos na rede básica e que muitas vezes vão necessitar de capacitações (P3).

[...] eu gosto da ideia do conteúdo geral, porque é interessante se atualizar e tirar as dúvidas, em relação a todo contexto da doença, mas eu concordo também que falta um pouco dessa especificidade (P5).

Rangel et al. (2011) consideram que a qualidade de um curso se refere as informações e não pela quantidade de inserção destas, sendo que a compreensão está relacionada com à interação teórico-prática que origina mudanças significativas e de abrangência específica.

[...] mais específica, seria interessante trazer um pouco dos problemas que a nossa atenção básica vivencia, os protocolos que sempre mudam, e são coisas que acabam travando o atendimento, acho que seria interessante abordar no curso essas dificuldades do dia a dia que você enfrenta, além da parte da doença em si (P1).

[...] eu concordo que poderia focar um pouco mais na parte específica, nas peculiaridades do município porque é direcionado para nossa rede, poderia se criar tópicos por exemplo dos pacientes paraguaios, os pacientes que não moram aqui mas que usam o serviço aqui, levantar essa discussão também, talvez de algumas unidades seja mais comum receber esses pacientes por causa da localização, mas em geral no município isso acaba sendo comum do paciente vir de fora, e o como manejar, como ter um olhar pra isso também seria interessante (P4).

No tocante à abordagem de conteúdo, os participantes destacaram a divisão dos conteúdos propostos e as diferentes estratégias de ensino:

[...] está realmente muito bem exposto e sucinto, além da seleção dos conteúdos estar com bom enfoque, uma vez que o assunto é muito abrangente (E6).

[...] a parte dos tópicos, as vezes você tem mais noção da fisiopatologia, mas você tem mais dúvida na transmissão, no tratamento, então você já vai direto e também por estar bem atualizado também (P5). 
[...] estratégico porque ele tem técnicas de estudo né que auxiliam na fixação do conteúdo, então ele é muito didático, então pra aquele aluno que aprende melhor com aula expositiva ou conteúdo teórico, ele tem reflexões, tem avaliações, eu achei ele bem estratégico (E2).

[...] eu gostei das atividades, de elaborar esquema (E3).

[...] agrega bastante porque tem a escrita, as atividades também que ajudam a fixação, linguagem acessivel, de entender e compreender, e os vídeos eles complementam bastante (E4).

[...] várias formas de ensino diferente para poder assimilar de forma mais eficaz (E5).

[...] o ponto forte era a gente ter mais de uma opção de conteúdo, além do vídeo, ter as escritas e tudo mais, bem dinâmico (P1).

[...] o melhor ponto a parte do fórum, onde tínhamos de elaborar um esquema pra enviar, então quando você tem que criar algo, criar algum protocolo, isso te força a pensar naquilo que você aprendeu, então ele estimula seu raciocínio crítico e fixa melhor aquele conteúdo que você recebeu, quando você precisa colocar esse conteúdo em prática (P2).

Coelho e Tedesco (2017) demonstram que há duas abordagens parecidas utilizando a EaD para EPS, uma ligada à construção colaborativa do conhecimento através de interações e a outra vinculada à autonomia dos aprendizes, uma interligando a outra.

Ao mesmo tempo, existem diversos meios de veicular informações, tais como por imagens, vídeos, exercícios, textos, jogos, entre outros, os quais são considerados objetos de aprendizagem de recurso educacional que possibilitam o autoaprendizado do participante (PEREIRA et al., 2016).

$\mathrm{Na}$ abordagem de avaliação do curso, os participantes quase que em sua totalidade concordaram com os métodos abordados e a possibilidade de verificarem os acertos e os erros para aprofundarem em tal conhecimento.

[...] eu gostei bastante das avaliações, dos outros métodos, como a parte do esquema que tem que fazer, o estudo de caso, isso é bem interessante porque a gente monta uma situação e já vai pensando em como isso vai ser solucionado né, ah! esse paciente chegou, falou tal coisa, como que a gente vai agir, então acho que as vezes esses outros métodos de avaliação são mais eficazes do que propriamente um monte de perguntas que vai apenas responder, eu acho que exigiu mais de mim, por exemplo, fazer o esquema do que responder as questões, então eu achei bem interessante mesclar essas avaliações (E1). 
[...] inserir a prática demandou um pouco mais de mim, e é bem legal quando tem estudo de caso, porque é uma outra forma de pensar né, você se contextualiza, é mais do que pensar a que remédio vai dar (E2).

[...] o estudo de caso faz a gente pensar mais em tudo né, e o que a gente faria na prática, é bem eficaz, o esquema também, o mapa mental ele ajuda bastante na fixação, facilita mais pra fixar o conteúdo, e o estudo de caso também porque força a gente a pensar o que a gente precisa e também a pensar nas ações, então é bem eficaz (E3).

[...] achei interessante outras formas de avaliação que nos faz pensar como enfermeiros mesmo e ações a serem realizadas (E5).

[...] na maioria das questões você tinha que prestar bastante atenção no curso pra responder né, não eram questões comuns, são coisas que realmente você tinha que dominar o conteúdo, o fato também de colocar as respostas do que você errou no final também gostei, pra você identificar onde foi seu erro, e estudar mais aquele conteúdo (P1).

[...] achei legal que tinha mais de uma forma de avaliação, além do fórum com as questões, tinham atividades que precisavam ser elaboradas e inseridas na plataforma, desse jeito a gente consegue atingir diferentes estilos de aprendizagem por exemplo, porque tem algumas pessoas que aprendem memorizando, outras construindo e no curso tinha mais de uma opção (P2).

Um dos participantes sugeriu novas possibilidades de métodos de avaliação que poderiam ser implementados, como o pré e pós-teste, e vídeos comentando as questões corrigidas para melhor adaptação e fixação de conteúdo:

[...] seria legal trabalhar com pré e pós-teste, pois esse método direciona o aluno para a avaliação, enfatizando os principais pontos e fixação dos aspectos mais importantes. Ainda, também seria interessante adotar ao final da avaliação um pequeno vídeo com a correção das perguntas comentadas, pois muitas vezes na correção das atividades comentadas que o aluno compreende e atesta melhor o assunto (E6).

No que se refere às percepções sobre a linguagem do curso, os participantes a julgaram estar de forma clara e adequada ao curso online:

[...] linguagem bem acessível pra nós da área da saúde e acho que para outra pessoa que for ler também será, uma linguagem bem clara e acessível (E1).

[...] simples, construtiva e prática (E4).

[...] bem entendivel e clara, fazendo-se bem compreensível para qualquer público (E6).

[...] está bem adequada (P2). 
[...] Achei de fácil entendimento, o que facilita para o aprendizado (P4).

[...] está clara a explicação nos textos, pra nós que somos profissionais está adequada (P6).

\section{Categoria IV - As potencialidades e debilidades encontradas no curso proposto}

Os participantes relataram que o curso apresentou muita qualidade e aplicável se comparado a outros já existentes, sendo de fácil compreensão e uso:

[...] gostei bastante da plataforma geral assim, o acesso está fácil, a linguagem está acessível e fácil, eu gostei bastante assim o conteúdo está bem explicado, é isso que precisamos, algo simples que seja acessivel e que a gente possa acessar em qualquer lugar que a gente tiver, em poder dar uma olhadinha pra relembrar pra estudar ainda, então eu gostei bastante da facilidade que tem a plataforma e também acrescentaria algo para as pessoas mais visuais (E2).

[...] Eu concordo e não tenho nenhum ponto a acrescentar, só parabenizar mesmo (E4).

[...] e eu não achei pontos negativos, acho que está ótimo assim (P6).

As falas dos participantes em meios aos desafios enfrentados no cenário epidemiológico e às constantes mudanças apontam ser necessário que os profissionais se mantenham atualizados, na medida em que o torna resolutivo e efetivo para a população, e cabe às instituições ofertar as demandas conforme as necessidades enfrentadas por esses profissionais em cada área (OLIVEIRA et al., 2019).

Alguns participantes indicaram as debilidades do curso que poderiam ser ajustadas e melhoradas, como torná-lo mais objetivo, acrescentando imagens, intérprete de libras, entre outros:

[...] seria interessante se por ventura pudesse ser feito intérprete de libras, seria legal para pessoa poder acompanhar aquilo que está sendo falado(P1).

[...] eu concordo também com essa parte de quem tem bastante texto no mesmo slide né, e poderia ter mais imagens, mais estudos, outras formas (P2).

[...] uma sugestão que eu deixo é de baixar as aulas teóricas, eu acho que se tiver como baixar é melhor porque as vezes a pessoa prefere imprimir para ler, tem muita gente que não gosta de ler no computador né, tem mais facilidade de ler no papel então se tivesse essa opção eu acho que ia ajudar (P3).

Ressalta-se que a internet facilita o processo de desenvolvimento social, científico e cultural do profissional de enfermagem, como se encontrou neste estudo e é corroborado 
por Cardoso et al. (2012), pois amplia e gera melhorias para a comunidade através da atualização de seus profissionais.

Houve ainda sugestão dos participantes para trabalhos futuros de associar o conteúdo a áreas específicas como, por exemplo, a TB na urgência e emergência:

[...] acho que seria legal para outros estudos colocar as complicações e a sintomatologia inicial de TB podem aparecer em outros setores, como na urgência e emergência, pois percebo no meu campo de supervisionado que quando aparece essas complicações em laudos de exames, temos a responsabilidade no encaminhamento e o manejo para minimizar os danos é falho (E6).

Destaca-se ser primordial que sejam disponibilizados recursos voltados à aprendizagem dos participantes dos cursos, tornando o ambiente de estudo fácil e de acesso múltiplo, possibilitando liberdade no processo de atualização do conhecimento e controle dos recursos utilizados (PETRA et al., 2015).

\section{CONSIDERAÇÕES FINAIS}

Considerando as características epidemiológicas da TB, é importante ressaltar sobre a não resolutividade dos serviços de saúde por inúmeros fatores, dentre eles a sua organização e capacitação dos profissionais, cujo aperfeiçoamento com a implementação do curso online, objeto deste estudo, e com diferentes metodologias e plataformas poderia contribuir neste processo, a fim de alcançar as metas de eliminação da doença e integralidade da assistência.

Neste estudo, observou-se que as alternativas de EPS poderiam ser trabalhadas como fontes de saber contributivas à almejada transversalidade do SUS, especialmente no que tange às enfermidades negligenciadas, como é o caso da TB, visando profissionais mais capacitados, críticos, proativos, autoavaliadores e autônomos, podendo desenvolver conceitos significativos para a sua práxis de assistência.

As limitações obtidas na realização deste estudo reportam-se ao tamanho da amostra, pois houve impossibilidade de reunir todos os estudantes e enfermeiros em um único horário e local tanto pela falta de disponibilidade dos mesmos quanto pela situação de pandemia do novo coronavírus com vistas a restrições de aglomerações por medidas de segurança, realizando-se assim GF online. 


\section{AGRADECIMENTO}

Ao professor Dr. Reinaldo Antônio Silva-Sobrinho, pela orientação deste trabalho em todas as suas etapas.

\section{REFERÊNCIAS}

AVELINO Carolina Costa Valcanti; COSTA, Lívia Cristina Scalon da; BUCHHORN, Soraia Matilde Marques; NOGUEIRA, Denismar Alves; GOYATÁ, Sueli Leiko Takamatsu. Teaching-learning evaluation on the ICNP ${ }^{\circledR}$ using virtual learning environment. Rev. Bras. Enferm., v. 70, n. 3, p. 630-7, 2017.

BRASIL. Secretaria de Vigilância em Saúde. Departamento de Vigilância das Doenças Transmissíveis. Manual de Recomendações para o Controle da Tuberculose no Brasil. Brasília: MS, 2019.

BRASIL. Ministério da Saúde. Secretaria de Vigilância em Saúde. Boletim Epidemiológico da Tuberculose. Brasília, n. esp., março. 2021.

BRASIL. Ministério da Saúde. Secretaria de Vigilância em Saúde. Boletim Epidemiológico da Tuberculose. Brasília, n. esp., março. 2020.

CAMPOS, Regiane Bezerra. Atenção à tuberculose em um município de tríplice fronteira internacional: o ensino como perspectiva para reorientação da assistência. Foz do Iguaçu: 2016. 73p. Dissertação (Mestrado em Ensino). Universidade Estadual do Oeste do Paraná.

CARDOSO, Ivana Macedo. "Rodas de Educação permanente" na Atenção Básica de Saúde: analisando contribuições. Saúde Soc., v. 21, n. supl. 1, p. 18-28, 2012.

COELHO, Willyans Garcia; TEDESCO, Patricia Cabral de Azevedo Restelli. A percepção do outro no ambiente virtual de aprendizagem: presença social e suas implicações para Educação a Distância. Rev. Bras. Educ., v. 22, n. 70, p. 609-24, 2017.

FALKENBERG, Mirian Benites; MENDES, Thais de Paula Lima; MORAES, Eliane Pedrozo de; SOUZA, Elza Maria de. Educação em saúde e educação na saúde: conceitos e implicações para a saúde coletiva. Cienc. Saúde Colet., v. 19, n. 3, p. 847-52, 2014.

FILATRO, Andrea Cristina; CAIRO, Sabrina. Produção de conteúdos educacionais. São Paulo: Saraiva, 2015.

KARPINSKI, Josiani Aparecida; DEL MOURO, Neirisleia Francisconi; CASTRO, Marcos; LARA, Luiz Fernando. Fatores críticos para o sucesso de um curso em EAD: a percepção dos acadêmicos. Avaliação (Campinas), v. 22, n. 2, p. 440-57, 2017.

MARCHISOTTI, Gustavo Guimarães; OLIVEIRA, Fátima Bayma de; LUKOSEVICIUS, Alessandro Prudêncio. The social representation of distance education from a Brazilian perspective. Ensaio: aval. pol. públ. Educ., v. 25, n. 96, p. 743-69, 2017.

MARTINS-MELO, Francisco Rogerlândio; LIMA, Mauricélia da Silveira; RAMOS JÚNIOR, Alberto Novaes; HEUKELBACK, Jorg; CAMPO, Márcia Oliveira Coelho. Modalidade de educação a distância na 
formação profissional em saúde da família: relato de experiência. Rev. Bras. Med. Fam. Comunidade, v. 9, n. 30, p.89-95, 2017.

MINAYO, Maria Cecília de Souza. Pesquisa social: teoria, método e criatividade. Rio de Janeiro: Vozes, 2003.

MINAYO, Maria Cecília de Souza. O desafio do conhecimento. São Paulo: Hucitec, 2007.

OLIVEIRA, Maíra Gussi de; SOUSA, Cibele Maria de; VARGAS, Cláudia Regina Merçon de; OLIVEIRA, Donizete Moreira de; LIMA, Maria da Glória; GUSSI, Maria Aparecida. Educação a distância como recurso para capacitação de Agentes Comunitários de Saúde para intervenções preventivas relacionadas ao álcool e outras drogas. Revista Eletrônica de Comunicação, Informação e Inovação em Saúde, v. 13, n. 1, p.48-61, 2019.

PEREIRA, Francisco Gilberto Fernandes; SILVA, Débora Valente da; SOUSA, Luciana Maria Oliveira de; FROTA, Natasha Marques. Construção de um aplicativo digital para o ensino de sinais vitais. Rev. Gaúcha Enferm., v. 37, n. 2, p. e59015, 2016.

PETRA, Thiago; MARCOLINO, Fernanda; CORSO, Aline; CAVALCANTI, Felipe. EaD colaborativa no sus: uma proposta da comunidade de práticas para os trabalhadores da saúde. Revista Teknos, v. 15, n. 2, p.49-59, 2017.

RANGEL, Elaine Maria Leite; MENDES, Isabel Amélia Costa; CÁRNIO, Evelin Capellari; ALVES, Leila Maria Marchi; CRISPIM, Juliane de Almeida; MAZZO, Alessandra; ANDRADE, Jesusmar Ximenes; TREVIZAN, Maria Auxiliadora; RANGEL, Alexandre Leite. Avaliação, por graduandos de enfermagem, de ambiente virtual de aprendizagem para ensino de fisiologia endócrina. Acta paul enferm., v. 24, n. 3, p.327-33, 2011.

SILVA-SOBRINHO, Reinaldo Antônio; ZILLY, Adriana; MONROE, Aline Aparecida; PINTO, Érika Simone Galvão; SILVA, Rosane Meire Munhak da; VILLA, Tereza Cristina Scatena. Ambivalência das ações de controle da tuberculose na atenção básica à saúde. Rev. Rene, v. 15, n. 4., p. 605-612, 2014.

WANDERLEY, Tatiana Peres Santana Porto; BATISTA, Mikael Henrique de Jesus; DUTRA JÚNIOR, Lázaro da Silva; SILVA, Valdirene Cassia. Docência em saúde: tempo de novas tecnologias da informação e comunicação. Revista Eletrônica de Comunicação, Informação e Inovação em Saúde, v. 12, n. 4, p. 488-501, 2018.

WORLD HEALTH ORGANIZATION. Global Tuberculosis Report 2019. Geneva: WHO; 2020.

WYSOCKI, Anneliese Domingues; PONCE, Maria Amélia Zanon; BRUNELLO, Maria Eugênia Firmino; BERALDO, Aline Ale; VENDRAMINI, Silvia Helena Figueiredo; SCATENA, Lúcia Marina; RUFFINONETTO, Antônio; VILLA, Tereza Cristina Scatena. Atenção Primária à Saúde e tuberculose: avaliação dos serviços. Rev. Bras. Epidemiol., v. 20, n. 1, p. 161-175, 2017.

Recebido em 19 de novembro de 2021.

Aprovado em 08 de dezembro de 2021.

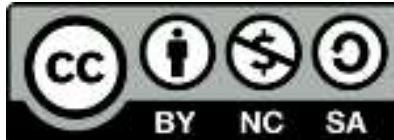

